\title{
EFEITOS DA REAÇÃO ÁLCALI-AGREGADO EM PILARES PAREDE DE CONCRETO
}

\author{
INICIAL DO NOME. SOBRENOME: E. MADUREIRA \\ Profissão: Professor \\ Instituição: UFRN \\ Estado; País: RioGrande do Norte; Brasil \\ e-mail: edmadurei@ct.ufrn.br
}

\section{RESUMO}

Os efeitos da Reação Álcali-Agregado, RAA, reportada por Stanton em 1940, repercutiram a partir da década de oitenta, quando foram constatados danos em estruturas de concreto, caracterizados pela fissuração localizada, ruína global e comprometimento da funcionalidade. Apesar do volume considerável de pesquisas realizadas sobre o assunto a modelagem voltada para a solução de problemas associados à referida patologia ainda necessita de aprimoramento. A simulação numérica representa recurso pertinente à avaliação dos efeitos decorrentes da RAA e à respectiva recuperação estrutural. O desenvolvimento, extensão e cronologia das avarias decorrentes de tal reação química, são influenciados por uma diversidade de fatores, a exemplo da umidade, da taxa de armadura do elemento estrutural, das tensões confinantes e da temperatura. $\mathrm{O}$ objetivo deste trabalho é a análise da influência da temperatura sobre o desempenho mecânico de membros de concreto afetados pelo efeito lesivo associado à RAA. Para o seu cumprimento é empregado "software" elaborado com base no método dos elementos finitos, relações constitutivas não lineares em estado plano de tensões para o concreto, e, um modelo termodinâmico para a avaliação de suas deformações por RAA. Os resultados obtidos indicaram o declínio da resistência inicial do concreto, resultando na diminuição da margem de segurança ao colapso, que em alguns casos foi deflagrado, e, para as temperaturas mais elevadas tal desencadeamento foi substancialmente antecipado.

Palavras-chave: simulação,numerica, temperatura, reação álcali-agregado.

\begin{abstract}
The Alkali-Aggregate Reaction effects reported by Stanton at 1940's year, resonated since the 80's decade, when damages in concrete structures were noted. Such damages were characterized by cracking, ruin and serviceability commitment. The modeling of the problem solution associated to the referring pathology still needs for improvement. The numerical simulation represents a suitable resource to the assessment of the effects derived from AAR and the respective structural recovering. The development of damages due to AAR, are influenced by factors as the moisture content and temperature. The aim of this paper is the analysis of temperature influence over the structural performance of concrete members affected by the AAR. To perform such a analysis a software drafted on the base of the finite element method, nonlinear constitutive relation in plane state of stresses for the concrete, and, a thermodynamic model to the assessment of the AAR swelling effect, are used. The obtained results revealed the concrete strength decrease due to AAR and so the safety margin to collapse reduction and, for the highest temperatures, the failure was anticipated. Keywords: numerical, simulation, temperature, alkali-aggregate reaction.
\end{abstract}

\section{INTRODUÇÃO}

A Reação Álcali-Agregado, RAA, transformação química envolvendo os álcalis do cimento e minerais dos agregados do concreto, foi reportada por Stanton em 1940 e despertou interesse no âmbito da Engenharia Civil a partir da década de oitenta, quando foram constatados danos em estruturas devidos a seus efeitos. As deficiências decorrentes incluíam fissuração local, ruína generalizada e comprometimento da funcionalidade.

Apesar do volume considerável de pesquisas a efetiva solução do problema ainda requer o aperfeiçoamento da teoria envolvendo a descrição do fenômeno. 
A simulação numérica constitui recurso fundamental para a estimativa das avarias provocados pela RAA, podendo auxiliar a tomada de decisões quanto à recuperação estrutural, e, servir de base para modificações de critérios de dimensionamento.

O desenvolvimento, a extensão e a cronologia de danos devidos à RAA são influenciados por uma diversidade de fatores condicionantes, tais como a umidade, a armadura do membro estrutural, as tensões confinantes e a temperatura.

O objetivo deste trabalho é a simulação numérica do desempenho mecânico de membros de concreto, afetados pela ação deletéria da Reação Álcali-Agregado.

Com vistas ao cumprimento de tal finalidade foi empregado "software" desenvolvido com base no método dos elementos finitos e relações constitutivas não lineares, e, um modelo termodinâmico de materiais porosos reativos, para a avaliação das deformações do concreto por RAA.

A análise foi realizada em estado plano de tensões sobre modelos diferenciados entre si pela taxa de armadura, pela intensidade do carregamento e pela temperatura.

\section{MODELAGEM}

Em problemas bi-dimensionais envolvendo análise estrutural, a matriz constitutiva referente ao modelo ortotrópico nãolinear é definida tomando-se por base, as deformações equivalentes, dadas por:

Os índices “ij" e "ij” representam as direções principais, os “ $\mathbf{D}_{\mathbf{i j}}$ " os elementos da matriz constitutiva, e os parâmetros $\boldsymbol{\varepsilon}_{\mathbf{i}}$ e $\boldsymbol{\varepsilon}_{\mathbf{j}}$ são as deformações segundo as direções principais. Para o concreto em compressão foram adotadas as relações de HOGNESTAD (1951):

onde " $\sigma_{\mathbf{p}}$ " é a tensão de pico do concreto, " $\varepsilon_{\mathbf{p}}$ " a deformação a ela correspondente, e, " $\varepsilon_{\mathbf{c u}}$ " a deformação limite de ruptura do material. Para o concreto em tração, adotou-se um modelo de fissuras distribuídas, cujas vantagens são considerar a continuidade do campo de deslocamentos, e, dispensar modificações de natureza topológica da malha de elementos finitos, no decorrer da análise. As tensões limite referentes ao concreto são definidas a partir da envoltória de KUPFER e GERSTLE (1973).

O comportamento do aço, por sua vez, foi considerado elástico perfeitamente plástico.

Para a simulação das expansões com o tempo, foi adotada a formulação termodinâmica para materiais porosos reativos proposta por CAPRA e SELLIER (2002) na forma:

sendo "A" o teor de álcalis consumido na reação; " $\mathbf{A}_{\mathbf{0}}$ " o valor de "A" ao início da expansão do concreto, e, " $\boldsymbol{\varepsilon}_{\mathbf{0}}$ " um fator que considera a defasagem entre os inícios da reação e da expansão da matriz de concreto. Este modelo é assim denominado porque correlaciona as deformações por RAA com a cinética da reação química. Para a modelagem da cinética da reação química, CAPRA e BOURNAZEL (1998), propuseram a equação:

para a qual “k(T)" é a constante cinética associada à lei de Arhenius, avaliada a partir de:

onde "E $\mathbf{E}_{\mathbf{a}}$ " é a energia de ativação, "R" a constante do gás ideal, "T" a temperatura e "k" a constante cinética. A solução da equação 4 é: 
Resultando, para a equação 3 no intervalo $\mathbf{A}>\mathbf{A}_{\mathbf{0}}$, a forma:

A influência das tensões confinantes foi quantificada conforme o critério proposto por CHARLWOOD (1994), expresso mediante:

onde " $\sigma_{i}$ " é a tensão principal na direção "i” em MPa, " $\varepsilon^{\text {RAA، }}$ a deformação confinada, " $\varepsilon_{u}^{R A A}$ " a deformação não confinada. " $\sigma_{\mathrm{L}}$ ", avaliada em $0,30 \mathrm{MPa}$, é a tensão abaixo da qual a expansão é livre. " $\sigma_{\max }$ “, da ordem de $8.0 \mathrm{MPa}$, é a tensão para a qual a deformação por RAA é nula, e "K" é a Inclinação da reta $\varepsilon$ x $\log \sigma$.

A influência da umidade é expressa mediante a curva de POOLE (1992):

onde "m" é um parâmetro de ajuste considerado igual a 8 e "H" é a umidade relativa.

Para exprimir a degradação do concreto por RAA, adotou-se formulação semelhante àquela proposta por PIETRUSZCZAC (1996), adaptada à marcha do andamento das deformações por RAA do modelo de CAPRA e SELLIER (2002), resultando:

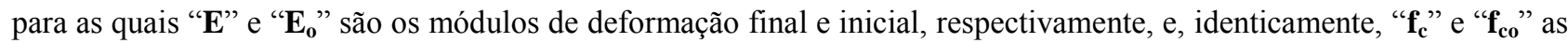
resistências à compressão. Os parâmetros " $\mathbf{A}_{\mathbf{E}}$ " e " $\mathbf{A}_{\mathbf{f}}$ " representam os fatores de intensidade da degradação do módulo de elasticidade e da resistência à compressão do concreto, respectivamente.

\section{SUPORTE COMPUTACIONAL}

Foi utilizado o "software" Análise Constitutiva Não Linear - ACNL, baseado no método dos elementos finitos e escrito em linguagem FORTRAN. O referido código computacional inclui em sua pauta algorítmica a formulação dos elementos isoparamétricos de aproximação quadrática, lineares L3, figura 1.a, voltados para a simulação das barras de aço, e planos quadriláteros Q8, figura 1.b, destinados à discretização da região da massa de concreto. As imagens referentes aos campos de deslocamentos foram geradas a partir do aplicativo NLPOS, PITANGUEIRA e PARENTE JR (1997), enquanto aquelas correspondentes aos campos de tensões foram produzidas a partir do aplicativo PROJECT1, elaborado em DELPH 10.2, MADUREIRA e SILVA (2013).

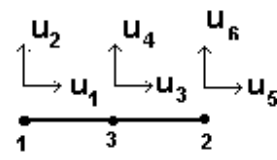

(a)

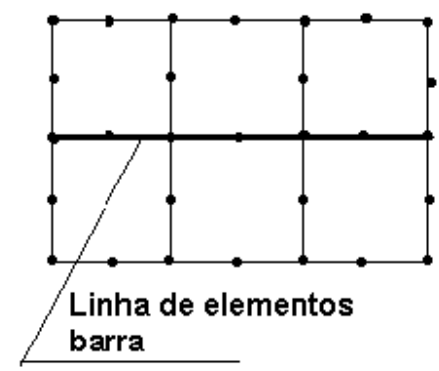

(b)

Figura 1: Elementos finitos: a - ) Lineares L3; b - ) Planos Q8

\section{VALIDAÇÃO DO PROGRAMA}

Para fins de validação, o programa foi utilizado para analisar, em estado plano de tensões, um pilar de concreto, de comprimento igual a 3,00 m e seção transversal retangular com dimensões de 0,25 m x 0,40 m, armado com quatro 
barras de $10 \mathrm{~mm}$ de diâmetro, submetido a carga axial de $400 \mathrm{kN}$. Os resultados de tal análise foram comparados com os seus correspondentes, obtidos em cálculo realizado a partir de modelo simplificado, elaborado com base nos postulados da mecânica dos sólidos, em estado de solicitação uniaxial, conforme RODRIGUES (2014), constatando-se boa concordância, tabela 1 .

Tabela 1 - Deslocamentos por RAA

\begin{tabular}{|c|c|c|c|}
\hline Tempo ( Dias ) & \multicolumn{3}{|c|}{ Deslocamentos $\left(10^{-3} \mathrm{~mm}\right.$ ) } \\
\hline & Modelo teórico & Modelo numérico & Diferença( \% ) \\
\hline 75 & 5,27 & 5,24 & 0,6 \\
\hline 150 & 9,90 & 9,80 & 1,0 \\
\hline 300 & 17,40 & 17,30 & 0,6 \\
\hline 600 & 28,30 & 28,10 & 0,7 \\
\hline 1200 & 41,20 & 40,90 & 0,7 \\
\hline 2500 & 54,00 & 53,60 & 0,7 \\
\hline 5000 & 62,90 & 62,50 & 0,6 \\
\hline 10000 & 68,60 & 68,10 & 0,7 \\
\hline
\end{tabular}

\section{MODELOS ANALISADOS}

Os modelos objeto de análise são pilares parede com dimensões conforme figura 2, onde estão representados graficamente com seu eixo longitudinal rotacionado de $90^{\circ}$ culminando por coincidir com a direção horizontal no plano da página (direção "x"). São constituídos de concreto C20, com módulo de deformação e coeficiente de Poisson de 21300,0 MPa e 0,17, respectivamente, armados com barras de aço CA-50, de módulo de elasticidade e limite de escoamento característico de 210000,0 MPa e 500,0 MPa, respectivamente, distribuídas conforme da figura 2. Foram analisados, em Estado Plano de Tensões, vinte e sete casos diferenciados entre si pela temperatura, pela taxa de armadura e pela intensidade da tensão solicitante, tabela 2.

Tabela 2 - Modelos estudados

\begin{tabular}{|c|c|c|c|c|c|c|c|}
\hline Caso & $\mathbf{T}\left({ }^{\circ} \mathbf{C}\right)$ & $\mathbf{A s}\left(\mathbf{c m}^{\mathbf{}}\right)$ & $\boldsymbol{\sigma}(\mathbf{M P a})$ & $\mathbf{C a s o}$ & $\mathbf{T}\left({ }^{\circ} \mathbf{C}\right)$ & $\mathbf{A s}\left(\mathbf{c m}^{2}\right)$ & $\boldsymbol{\sigma}(\mathbf{M P a})$ \\
\hline $\mathbf{1}$ & 20 & 12,80 & 2,00 & $\mathbf{1 5}$ & 50 & 20,00 & 8,00 \\
\hline $\mathbf{2}$ & 20 & 12,80 & 4,00 & $\mathbf{1 6}$ & 50 & 32,00 & 2,00 \\
\hline $\mathbf{3}$ & 20 & 12,80 & 8,00 & $\mathbf{1 7}$ & 50 & 32,00 & 4,00 \\
\hline $\mathbf{4}$ & 20 & 20,00 & 2,00 & $\mathbf{1 8}$ & 50 & 32,00 & 8,00 \\
\hline $\mathbf{5}$ & 20 & 20,00 & 4,00 & $\mathbf{1 9}$ & 100 & 12,80 & 2,00 \\
\hline $\mathbf{6}$ & 20 & 20,00 & 8,00 & $\mathbf{2 0}$ & 100 & 12,80 & 4,00 \\
\hline $\mathbf{7}$ & 20 & 32,00 & 2,00 & $\mathbf{2 1}$ & 100 & 12,80 & 8,00 \\
\hline $\mathbf{8}$ & 20 & 32,00 & 4,00 & $\mathbf{2 2}$ & 100 & 20,00 & 2,00 \\
\hline $\mathbf{9}$ & 20 & 32,00 & 8,00 & $\mathbf{2 3}$ & 100 & 20,00 & 4,00 \\
\hline $\mathbf{1 0}$ & 50 & 12,80 & 2,00 & $\mathbf{2 4}$ & 100 & 20,00 & 8,00 \\
\hline $\mathbf{1 1}$ & 50 & 12,80 & 4,00 & $\mathbf{2 5}$ & 100 & 32,00 & 2,00 \\
\hline $\mathbf{1 2}$ & 50 & 12,80 & 8,00 & $\mathbf{2 6}$ & 100 & 32,00 & 4,00 \\
\hline $\mathbf{1 3}$ & 50 & 20,00 & 2,00 & $\mathbf{2 7}$ & 100 & 32,00 & 8,00 \\
\hline $\mathbf{1 4}$ & 50 & 20,00 & 4,00 & & & & \\
\hline
\end{tabular}

Aproveitando sua simetria o domínio do problema no plano "xy" foi definido conforme ilustrado na figura 2 que, uma vez discretizado mediante elementos de dimensão igual a $0,10 \mathrm{~m}$, resultou em malha com 240 elementos finitos planos e 120 elementos unidimensionais.

A energia de ativação da reação qumica, a RAA, foi estimada em $8500 \mathrm{~m}^{3} \cdot \mathrm{Pa}^{\mathrm{m}} \mathrm{mol}^{-1}$, Ghanem et al,. (2010). Para o número de Avogadro adotou-se o valor 6,02214129 x $10^{23} \mathrm{~mol}^{-1}$. Os fatores de intensidade da degradação do módulo de elasticidade, e, da resistência à compressão do concreto foram fixados com base nos resultados experimentais de Swamy e Al-Asali (1988), resultando $\mathbf{A}_{\mathbf{E}}=0,3$ e $\mathbf{A}_{\mathbf{f}}=0,4$, respectivamente. 
O pilar foi carregado aos 28 dias de idade do concreto mediante uma ação uniformemente distribuída na área da seção transversal de seu topo, figura 4, prescrevendo-se para prazo limite de análise do fenômeno o lapso de 8000 dias.

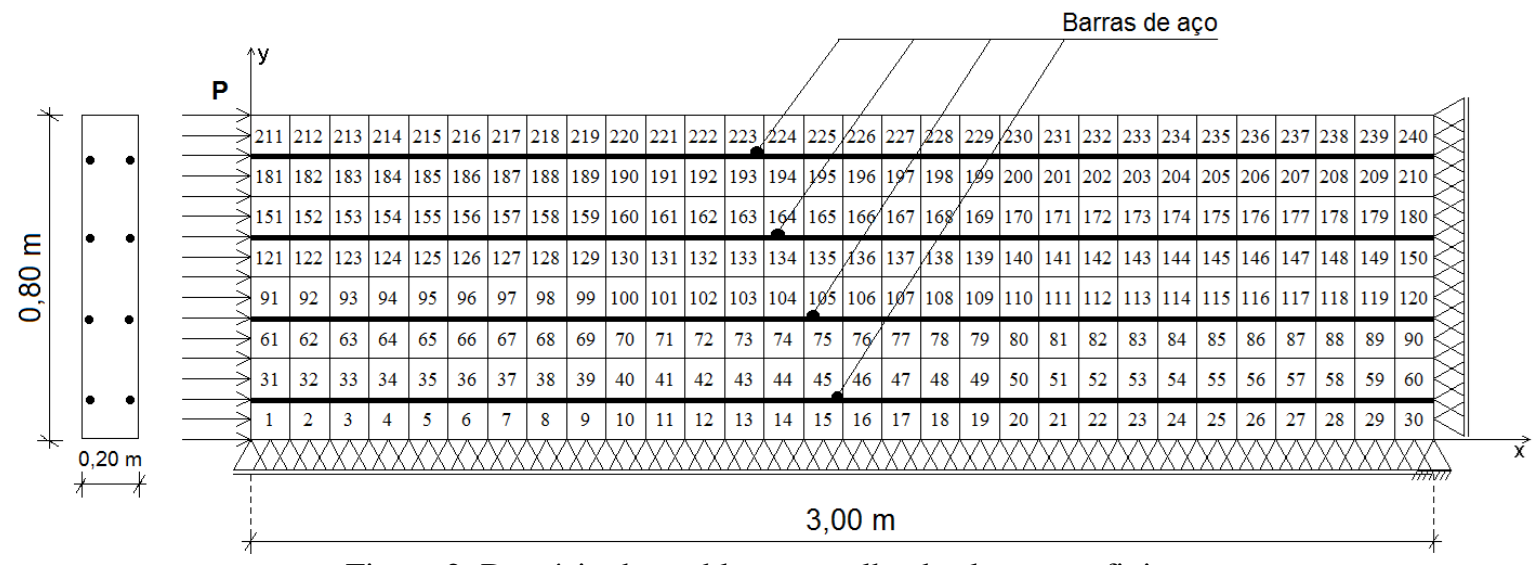

Figura 2: Domínio do problema e malha de elementos finitos

\section{RESULTADOS}

Os resultados obtidos indicaram que, para os casos "1", “4”, “7”, “10”, “13”, "16", "19”, “22” e "25”, cuja tensão de compressão na direção " $\mathrm{x}$ " é de 2,0 MPa, os deslocamentos e as tensões referentes ao carregamento solicitante, apresentaram-se conforme os campos das Figuras 3 e 4 , respectivamente. O encurtamento do pilar foi de 0,30 mm. Para os casos referentes à tensão de 4,0 e 8,0 MPa, tabela 3, os encurtamentos foram de 0,60 e 1,30 mm, respectivamente.
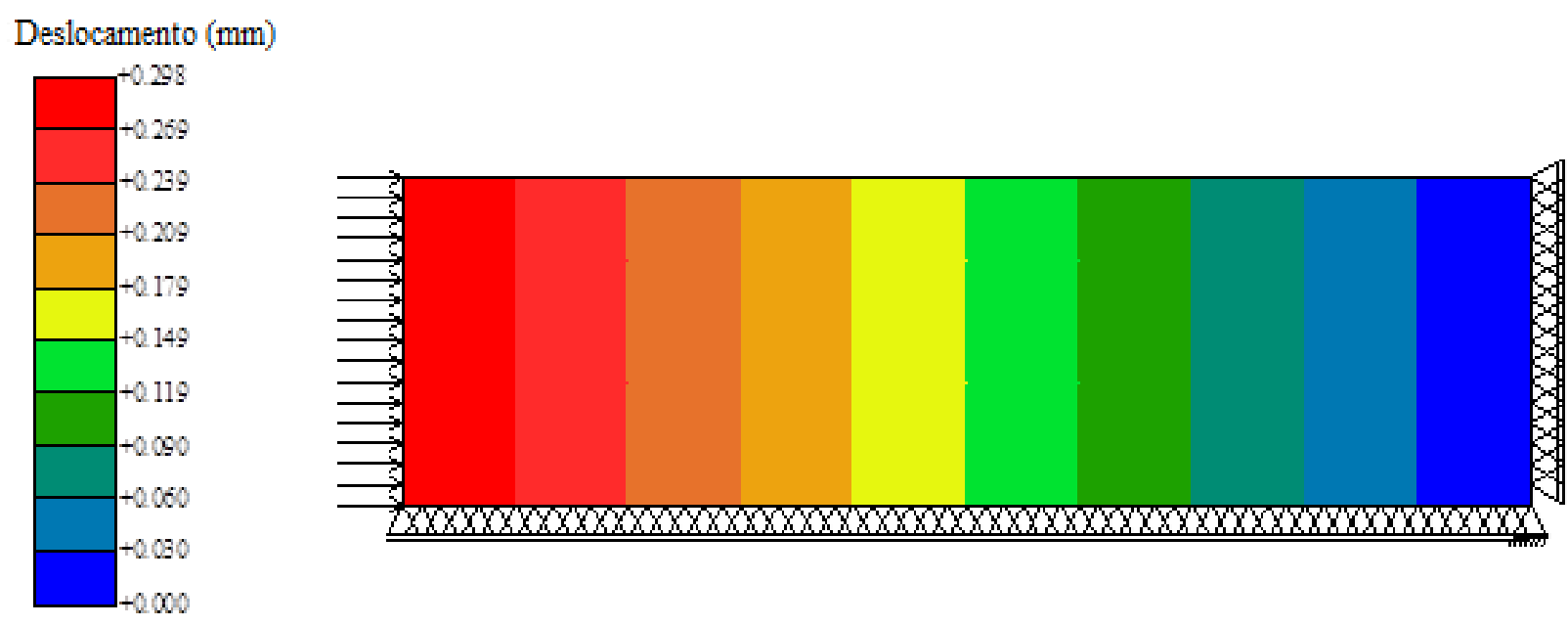

Figura 3: Deslocamentos na direção "x" imediatos ao carregamento para o caso "1"
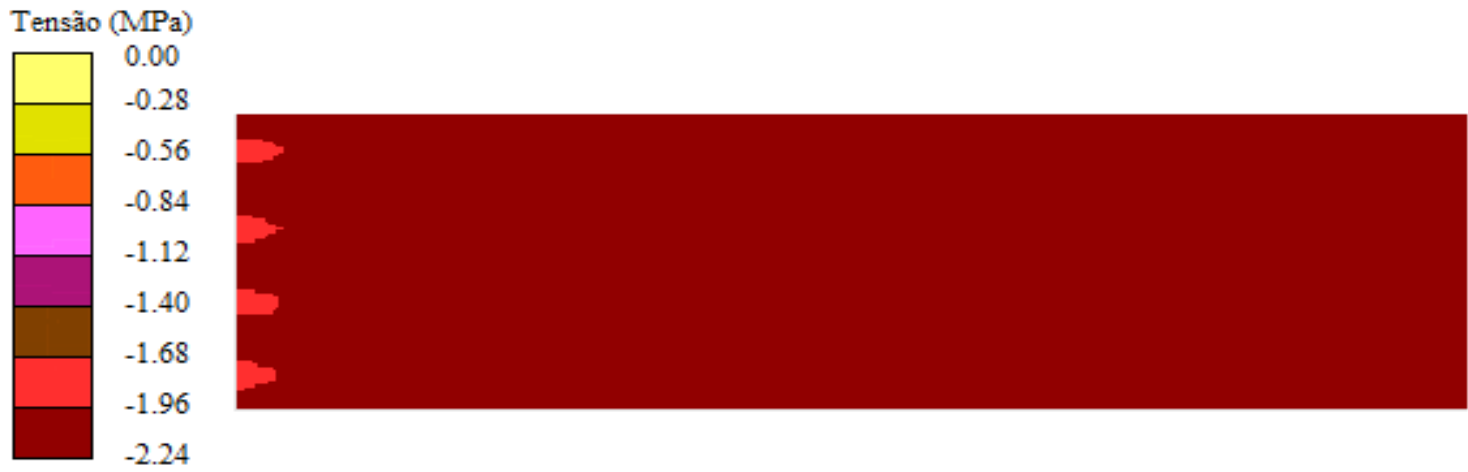

Figura 4: Tensões na direção " $x "$ imediatas ao carregamento para o caso "7" 
Uma vez deflagrado o efeito expansivo da RAA, os pilares parede alongaram-se progressivamente conforme as curvas da figura 5, observando-se três feixes distintos de linhas, ressaltando que as expansões são maiores para tensões de compressão de menor intensidade. Constata-se, inclusive, que para os casos “1”, "4", “7”, “10", “13", "16", "19”, “22" e " 25 ", o processo já havia se estabilizado aos 8000 dias de idade do concreto, quando apresentou distribuição conforme o campo da figura 6 , resultando alongamentos de $0,27 \mathrm{~mm}$, que representam $90 \%$ da contração no instante imediato ao carregamento, tabela 3 .

Os modelos dos casos "2", “5”, “8”, “11”, “14", “17”, “20”, “23” e “26”, atingiram alongamentos, da ordem de 0,13 $\mathrm{mm}$ aos 8000 dias, e, portanto, de $21 \%$ da contração imediata ao carregamento. Para os demais modelos, as expansões em decorrência da RAA foram praticamente nulas.

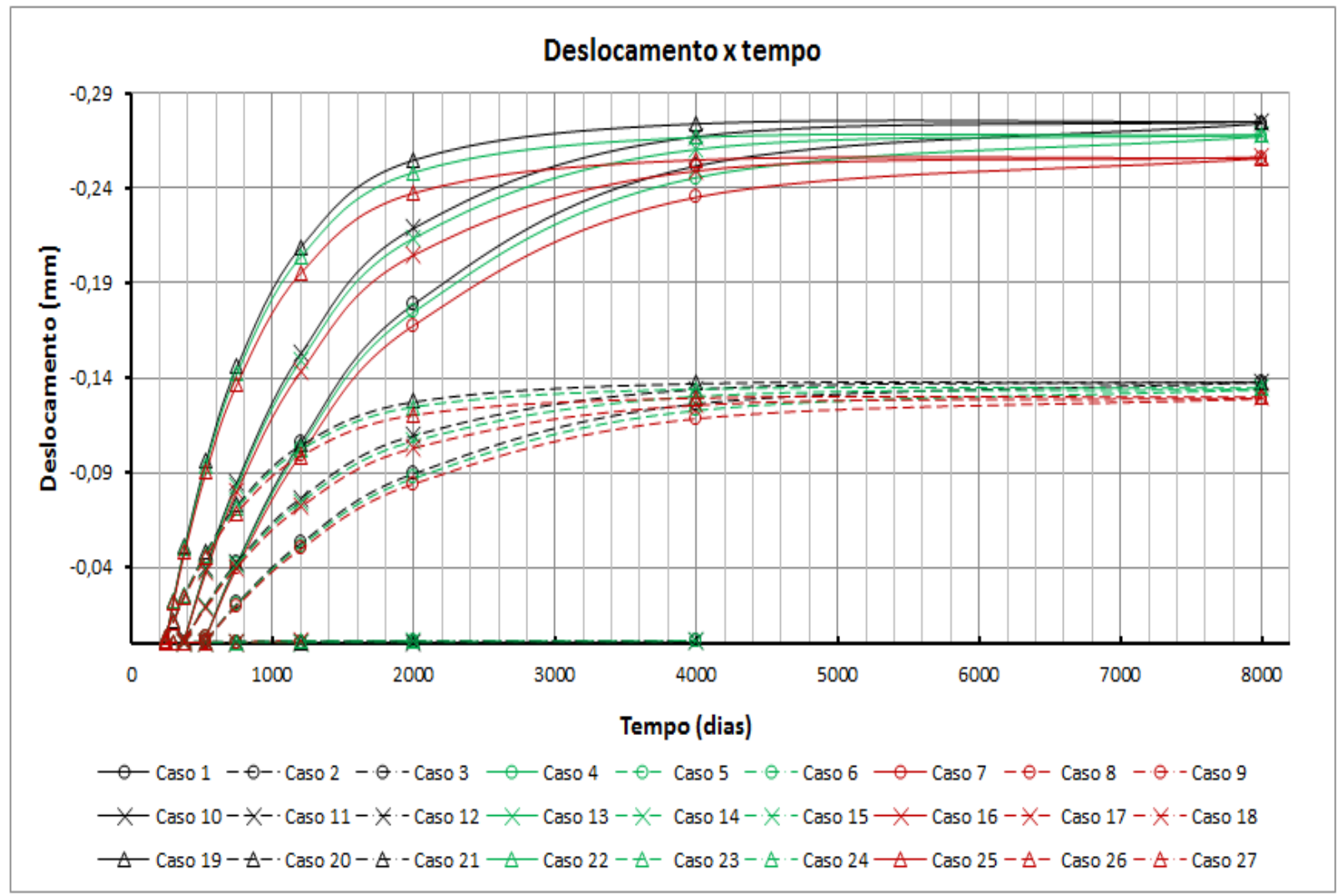

Figura 5: Deslocamentos na direção "x" devidas à RAA
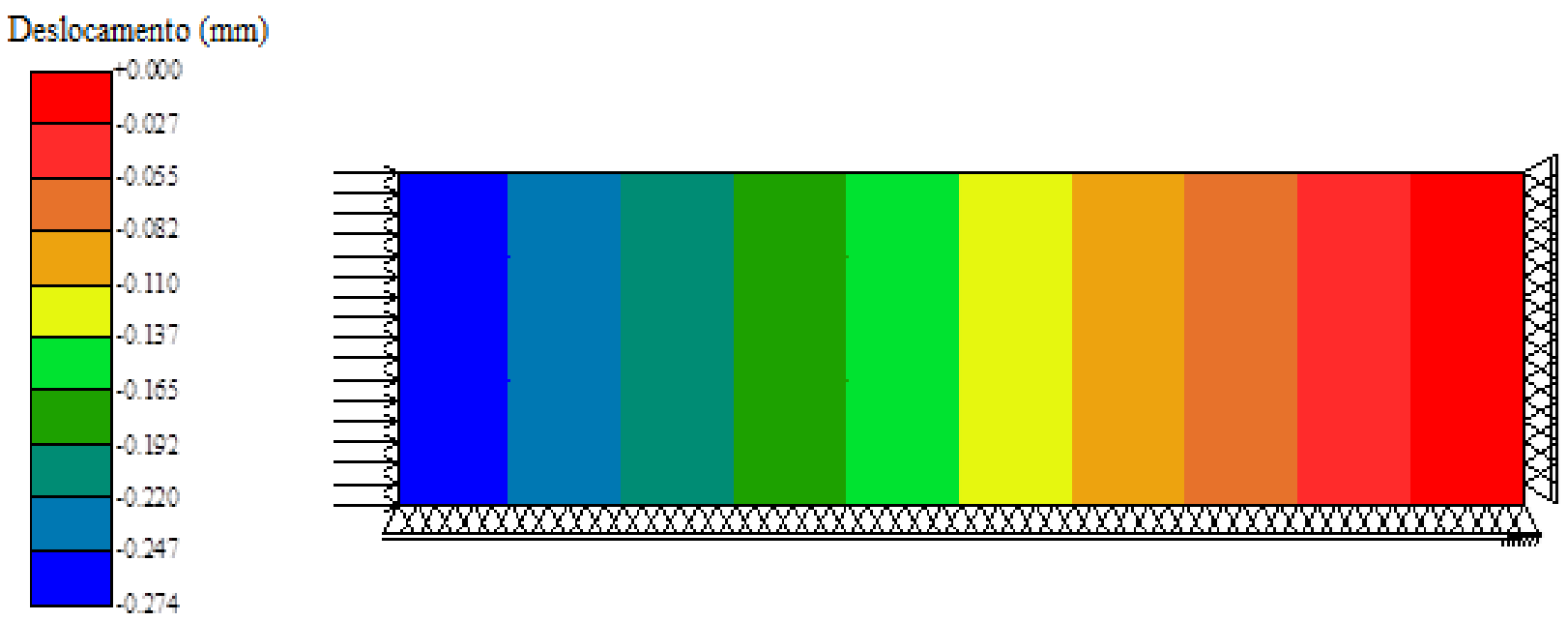

Figura 6: Deslocamentos na direção "x" por RAA aos 8000 dias de idade para o caso "1" 
Verificou-se ainda que, para os casos "1","2", “4", “5", “7" e "8", referentes à temperatura de $20^{\circ} \mathrm{C}$, as expansões por RAA só iniciaram-se aos 510 dias de idade do concreto. Para os casos "10", "11”, "13”, "14”, "16" e "17”, por sua vez, referentes à temperatura de $50^{\circ} \mathrm{C}$, o início se deu aos 370 dias, e, para os casos "19", "20”, "22", "23", "25” e “26", relativos à temperatura de $100^{\circ} \mathrm{C}$, aos 240 dias, figura 7.

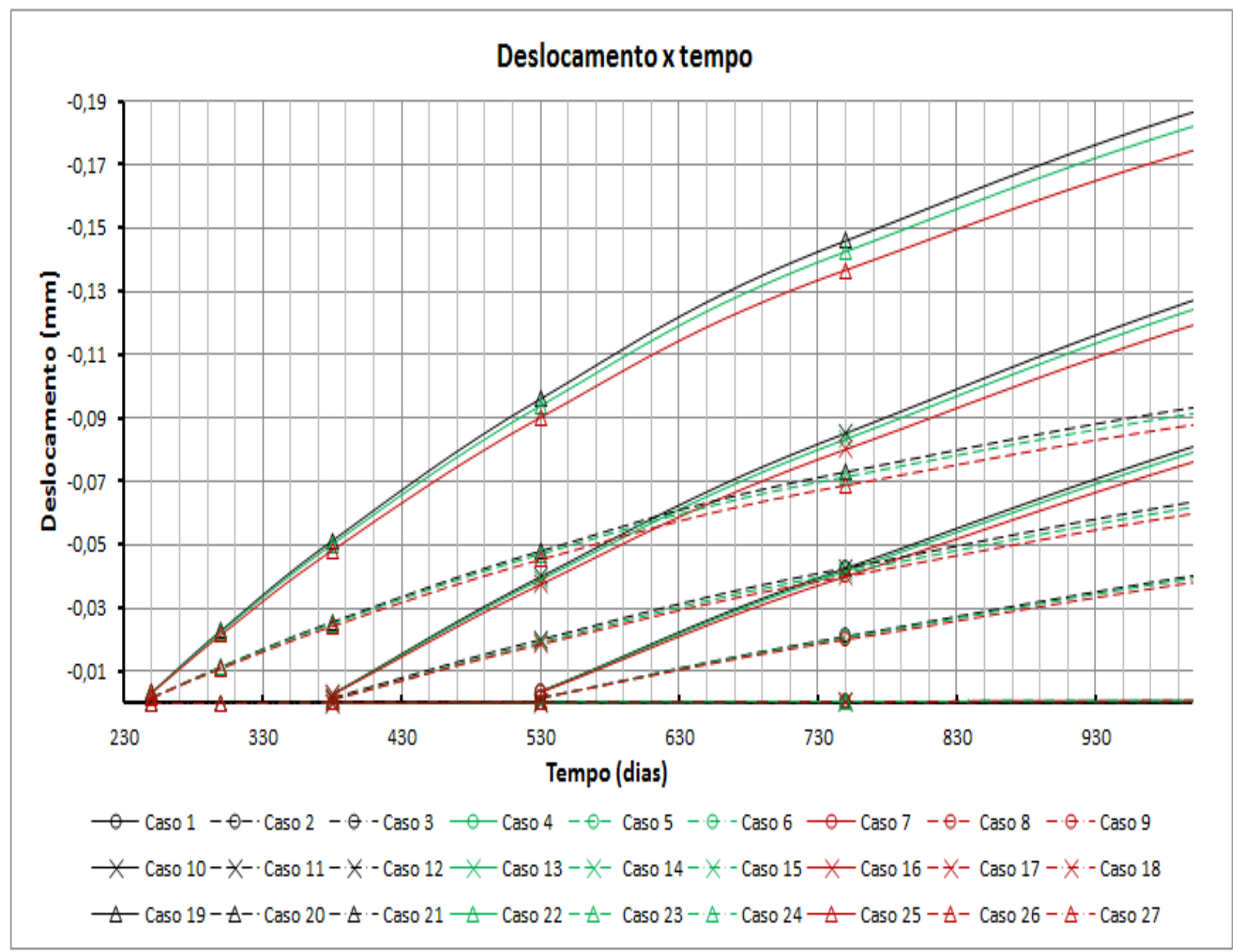

Figura 7: Início dos deslocamentos na direção "x" devidas à RAA

Examinando-se os índices da tabela 3 constata-se que, para os casos “3”, “6”, “9”, "12", “15”, “18”, "21", "24” e “27”, as tensões na armadura mantiveram-se constantes, com um valor em torno de $92 \mathrm{MPa}$, como consequência natural do fato de os deslocamentos por RAA, serem nulos para esses casos. Com respeito aos casos "2", "5", "8", “11", "14", “17" "20", "23” e "26" houve redução na intensidade dessas tensões de $43 \mathrm{MPa}$ para $33 \mathrm{MPa}$, e, para os casos "1", “4”, “7”, “10", "13", “16" “19”, "22” e "25” a redução foi de $21 \mathrm{MPa}$ para $2 \mathrm{MPa}$, tabela 3.

Apesar de as expansões por RAA terem promovido pouca variação nos campos de tensões na massa de concreto, o efeito da reação promoveu variações significativas nas Tensões Normalizadas de Compressão, induzidas pelo declínio da resistência do concreto, figura 8.

Para os casos “1”, “4”, “7”, “10”, “13”, “16”, “19”, “22” e “25”, o aumento registrado foi discreto perdurando, aos 8000 dias de idade do concreto, margem de segurança considerável. Nos casos “2”, “5”, "8”, “11”, “14”, “17”, “20", “23” e "26" o acréscimo foi de 0,20 para em torno de 0,67 aos 8000 dias de idade do concreto, perdurando uma margem de segurança em torno de 33\%. Para os casos “3”, "12" e "21", para as quais a tensão solicitante é de 8,0 MPa e a área de armadura de aço é menor, o aumento da Tensão de Compressão Normalizada foi de 0,40 para 1,0 determinando condição de elevada probabilidade de ruína por esmagamento do concreto, já aos 1600, 2500 e 3600 dias de idade do concreto, respectivamente. 
Tabela 3 - Resumo de deslocamentos e tensões para todos os casos de análise

\begin{tabular}{|c|c|c|c|c|c|c|}
\hline \multirow[t]{2}{*}{ Caso } & \multicolumn{2}{|c|}{ Deslocamento (mm) } & \multicolumn{2}{|c|}{ Tensão no Concreto (MPa) } & \multicolumn{2}{|c|}{ Tensão na Armadura (MPa) } \\
\hline & Carregamento & RAA & Carregamento & RAA & Carregamento & RAA \\
\hline 1 & 0,30 & $-0,27$ & $-2,00$ & $-2,07$ & $-20,85$ & $-1,66$ \\
\hline 2 & 0,61 & $-0,14$ & $-4,00$ & $-4,04$ & $-42,89$ & $-33,31$ \\
\hline 3 & 1,30 & 0,00 & $-8,00$ & $-8,00$ & $-91,55$ & $-91,55$ \\
\hline 4 & 0,30 & $-0,27$ & $-2,00$ & $-2,11$ & $-20,83$ & $-2,11$ \\
\hline 5 & 0,61 & $-0,13$ & $-4,00$ & $-4,06$ & $-42,90$ & $-33,57$ \\
\hline 6 & 1,30 & 0,00 & $-8,00$ & $-8,00$ & $-91,53$ & $-91,53$ \\
\hline 7 & 0,30 & $-0,26$ & $-2,00$ & $-2,17$ & $-20,89$ & $-2,98$ \\
\hline 8 & 0,61 & $-0,13$ & $-4,00$ & $-4,08$ & $-42,82$ & $-33,83$ \\
\hline 9 & 1,30 & 0,00 & $-8,00$ & $-8,00$ & $-91,49$ & $-91,50$ \\
\hline 10 & 0,30 & $-0,28$ & $-2,00$ & $-2,08$ & $-20,85$ & $-1,58$ \\
\hline 11 & 0,61 & $-0,14$ & $-4,00$ & $-4,04$ & $-42,89$ & $-33,27$ \\
\hline 12 & 1,30 & 0,00 & $-8,00$ & $-8,00$ & $-91,55$ & $-91,55$ \\
\hline 13 & 0,30 & $-0,27$ & $-2,00$ & $-2,11$ & $-20,83$ & $-2,05$ \\
\hline 14 & 0,61 & $-0,13$ & $-4,00$ & $-4,06$ & $-42,90$ & $-33,53$ \\
\hline 15 & 1,30 & 0,00 & $-8,00$ & $-8,00$ & $-91,53$ & $-91,53$ \\
\hline 16 & 0,30 & $-0,26$ & $-2,00$ & $-2,18$ & $-20,89$ & $-2,93$ \\
\hline 17 & 0,61 & $-0,13$ & $-4,00$ & $-4,08$ & $-42,82$ & $-33,80$ \\
\hline 18 & 1,30 & 0,00 & $-8,00$ & $-8,00$ & $-91,49$ & $-91,50$ \\
\hline 19 & 0,30 & $-0,27$ & $-2,00$ & $-2,08$ & $-20,85$ & $-1,59$ \\
\hline 20 & 0,61 & $-0,14$ & $-4,00$ & $-4,04$ & $-42,89$ & $-33,27$ \\
\hline 21 & 1,30 & 0,00 & $-8,00$ & $-8,00$ & $-91,55$ & $-91,55$ \\
\hline 22 & 0,30 & $-0,27$ & $-2,00$ & $-2,12$ & $-20,83$ & $-2,07$ \\
\hline 23 & 0,61 & $-0,13$ & $-4,00$ & $-4,06$ & $-42,90$ & $-33,53$ \\
\hline 24 & 1,30 & 0,00 & $-8,00$ & $-8,00$ & $-91,53$ & $-91,53$ \\
\hline 25 & 0,30 & $-0,26$ & $-2,00$ & $-2,18$ & $-20,89$ & $-2,96$ \\
\hline 26 & 0,61 & $-0,13$ & $-4,00$ & $-4,09$ & $-42,82$ & $-33,81$ \\
\hline 27 & 1,30 & 0,00 & $-8,00$ & $-8,00$ & $-91,49$ & $-91,50$ \\
\hline
\end{tabular}

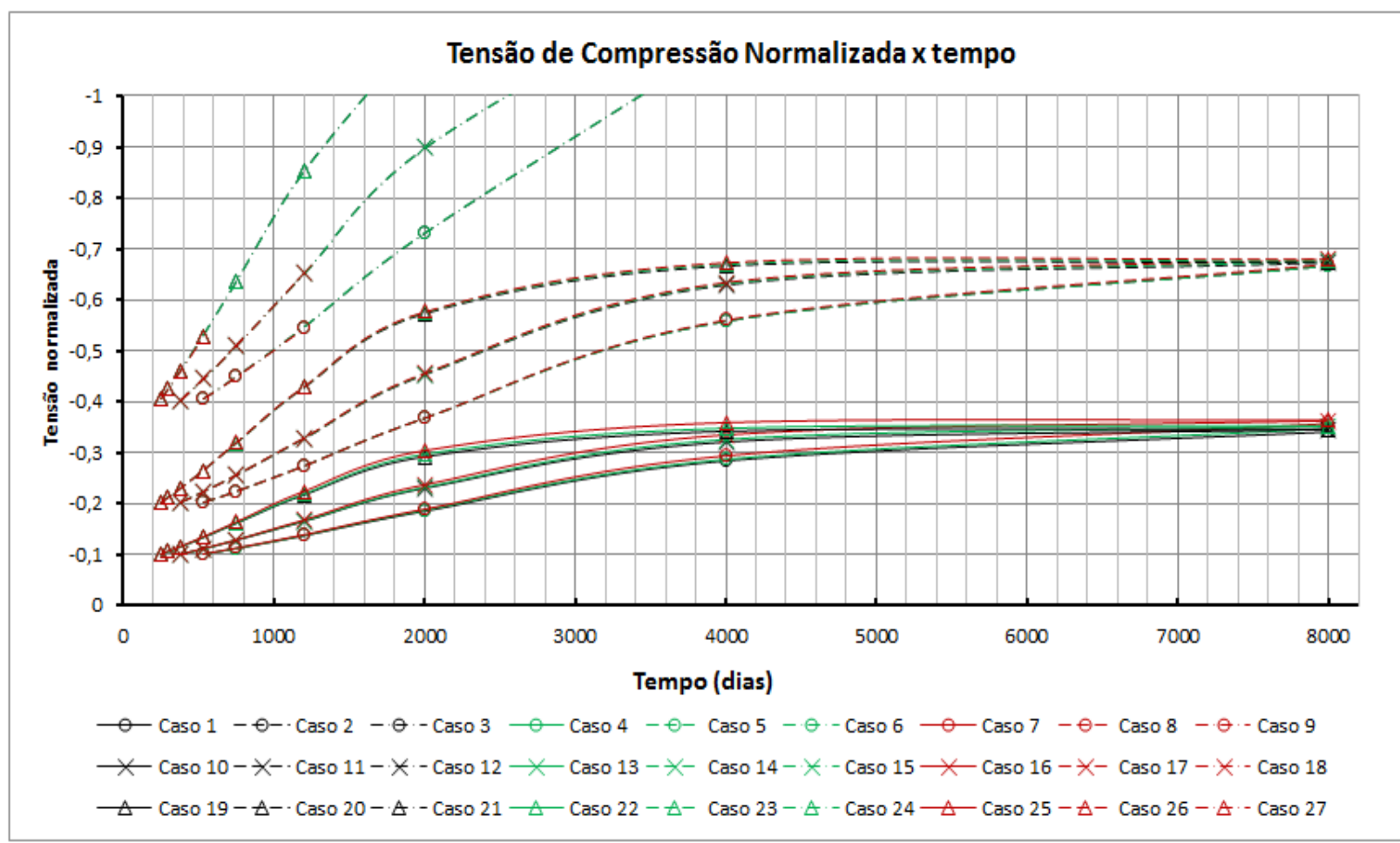

Figura 8: Evolução da Tensão de Compressão Normalizada com a RAA 


\section{CONCLUSÕES}

Este trabalho se refere à análise do desempenho de pilar parede de concreto armado afetado pela Reação ÁlcaliAgregado, a RAA, a partir da Aproximação por Elementos Finitos e Formulação Ortotrópica não Linear, sobre um Modelo Termodinâmico de Materiais Porosos.

A análise foi realizada sobre vinte e sete casos diferenciados entre si pela temperatura da massa do concreto, pela taxa de armadura e pelo carregamento solicitante, com vistas a avaliar a influência desses parâmetros no desempenho mecânico do elemento estrutural em estudo, cujo concreto de constituição esteja afetado pela reação em destaque.

Os resultados obtidos corroboraram o esperado efeito de contenção das deformações por RAA exercido pela tensão de compressão uma vez que quanto maior sua intensidade tanto menores foram as magnitudes da expansão do membro estrutural analisado.

Os campos de tensões apresentaram pouca variação no transcorrer do processo expansivo devido à RAA, o que deve estar associado ao fato de os deslocamentos terem apresentado baixa magnitude, o elemento estrutural ser externamente isostático e a hiperestaticidade interna do conjunto ser insuficiente para provocar redistribuição expressiva de tensões.

A ação deletéria da RAA sobre a massa do concreto foi evidente, haja vista seu efeito de empobrecimento do desempenho mecânico do material, uma vez que, promoveu a redução de sua resistência. Este efeito assumiu relevância, sobretudo, para os casos de maior tensão inicial, pois, embora a tensão de compressão tenha apresentado pouca variabilidade, sua versão normalizada em relação à tensão de pico do material atingiu valores iguais à unidade, caracterizando condição de elevada probabilidade de ruína.

Ressalta-se que nos casos para os quais se prescreveu as temperaturas mais elevadas, as expansões por RAA foram desencadeadas em idades mais jovens do concreto, sem contar que a ruína do elemento estrutural foi antecipada. Tal realidade induz a necessidade de fixarem-se diferentes periodicidades para as intervenções corretivas, voltadas à preservação do bom desempenho mecânico do elemento estrutural, e até mesmo reduzir a previsão legal de sua vida útil. Por outro lado, os valores finais dos deslocamentos por RAA, mostraram-se indiferentes à magnitude da temperatura, corroborando que o efeito catalisador a ela associado restringe-se, exclusivamente, à cinética da reação, e, em consequência, exerce, tão somente, o efeito acelerador.

Por outro lado, apesar de se esperar que, a taxas de armaduras maiores correspondessem menores expansões por RAA, em face de seu presumido efeito de contenção, esta tendência não ficou evidente. Tal realidade pode ser atribuída à propensão das armaduras passivas, de taxas maiores, atraírem para si esforços normais mais elevados, resultando em tensões de compressão menores no concreto, favorecendo a ampliação das deformações devidas ao efeito em estudo.

\section{REFERÊNCIAS}

CAPRA, B. E BOURNAZEL, J. P..Modelling of Induced Mechanical effects of Álkali-Agregate Reactions. Cement and Concrete Research, Vol. 28, N.2, pp 251-260, 1998.

CAPRA, B. e SELLIER, A.. Ortotropic Modelling of Alkali-Aggregate Reaction in Concrete Structures: Numerical Simulations. Mechanics of Materials, 2002.

CHARLWOOD, R.G. A Review of Alkali-aggregate Reaction in Hydro-electric Plants and Dams. Hydropower Dams, n 1, pp 73-80, 1994.

GHANEM, H., ZOLLINGER, D. E LYTTON, R. predicting asr aggregate reactivity in terms of its activation energy. construction and building materials, vol. 24, pp. 1101-1108, 2010.

HOGNESTAD, E.. A Study of Combined Bending and Axial Load in Reinforced Concrete Members. Bolletin $n$. 399, Engineering Experiment Station, University of Illinois, Urbana, Illinóis, Vol. 49, n 22, 1951.

KUPFER, H.B. e GERSTLE, K.H.. Behaviour of Concrete under Biaxial Stresses. Journal of Engineering Mechanics, Vol. 99, n. 4, pp. 853-866, 1973. 
MADUREIRA, E.L. e SILVA, A.L.A. PROJECT1 - Programa para visualização de campos de tensões resultantes de analises não lineares de modelos bidimensionais de elementos finitos. Versão 1.0, Rio Grande do Norte: DEC/UFRN, 2013.

PIETRUSZCZAC, S.. On the Mechanical Behaviour of Concrete Subjected to Alkali-Aggregate Reaction. Computers \& Structures. Vol. 58, n. 6, pp 1093-1097,1996.

PITANGUEIRA, R. L. S. e PARENTE JR., E. NLPOS - Programa para visualização de resultados de analises não lineares de modelos bidimensionais de elementos finitos. Versão 1.0, Rio de Janeiro: DEC/PUC-Rio, 1997.

POOLE, A.B. The Alkali-silica Reaction in Concrete. Ed Swamy, Blackie, London, 1992.

RODRIGUES, E.C. Análise Numérica do Efeito de Fatores Influentes da Reação Álcali-Agregado no Desempenho de Estruturas de Concreto. Dissertação de Mestrado. Programa de Pós-Graduação em Engenharia Civil. Universidade Federal do Rio Grande do Norte, 2014.

SWAMY, R. N. , e AL-ASALI, M. M. Engineering Properties of Concrete Affected by Alkali-Silica Reaction. ACI Materials Journal, September/October 1988, pp.367-374, 1988. 